\title{
Análise ultrassonográfica do quadríceps femoral de pacientes críticos sob ventilação mecânica
}

\section{Ultrasound analysis of the quadriceps femoris of critically ill patients on mechanical ventilation}

\author{
Roberta Virginia Silva Alves de Lima ${ }^{1}$ \\ Júlia Sales Fernandes ${ }^{2}$ \\ Auriwerton Santos Lucena de Sousa ${ }^{3}$ \\ Dyego Tavares de Lima ${ }^{4}$ \\ Anderson Ferreira Rodrigues 5 \\ Rafaela Pedrosa ${ }^{6}$ \\ José Heriston de Morais Lima? \\ Eduardo Eriko Tenório de França ${ }^{8}$
}

Número do Parecer do Comitê de Ética: 3.283.172

Endereço para correspondência:

Eduardo Eriko Tenório de França.

Universidade Federal da Paraíba. Conj. Pres. Castelo Branco III, João Pessoa/PB, Brasil. 58033-455.

Telefone: (83) 32167183

edueriko@hotmail.com

${ }^{1}$ Bacharel em Fisioterapia, Universidade federal da Paraíba,

João Pessoa/PB/Brasil. robertaavsilva@gmail.com

${ }^{2}$ Bacharel em Fisioterapia, Universidade Federal da Paraíba,

João Pessoa/ PB/Brasil. juliasalesf@hotmail.com

${ }^{3}$ Especialista, Hospital Universitário Lauro Wanderley,

João Pessoa/PB/Brasil. auriwerton.lucena@hotmail.com

${ }^{4}$ Especialista, Hospital Universitário Lauro Wanderley,

João Pessoa/PB/Brasil. dyego.fisio@hotmail.com

${ }^{5}$ Mestre, Hospital Universitário Lauro Wanderley,

João Pessoa/PB/Brasil. andfisio@hotmail.com

${ }^{6}$ Doutora, Departamento de Fisioterapia, Universidade Federal da Paraíba, João Pessoa/PB/Brasil.

rafaela_pedrosa@yahoo.com.br

${ }^{7}$ Pós-Doutor, Departamento de Fisioterapia, Universidade Federal da Paraíba, João Pessoa/PB/Brasil.

joseheristonlima@yahoo.com.br

${ }^{8}$ Doutor, Departamento de Fisioterapia e Programa de Pós-graduação em Fisioterapia, Universidade Federal da Paraíba, João Pessoa/PB/Brasil edueriko@hotmail.com

\section{Resumo}

Introdução: Devido aos diversos danos gerados pela imobilidade no leito, fazse necessária uma avaliação precoce do estado muscular dos pacientes críticos. Objetivo: Comparar as imagens ultrassonográficas e correlacionar a espessura muscular com a ecointensidade do músculo reto femoral (RF) e vasto intermédio (VI) em 48 horas e com 7 dias de ventilação mecânica invasiva (VMI).

Métodos: Trata-se de um estudo observacional prospectivo, com 53 imagens obtidas numa Unidade de Terapia Intensiva.

Resultados: Houve redução na espessura muscular de RF, VI e espessura total. Observamos uma correlação negativa entre a espessura muscular e ecointensidade do RF nas primeiras 48 horas e 7 dias após a VMI. Já em relação ao VI, não observamos associação da espessura do VI com a ecointensidade nesse mesmo período.

Conclusão: Evidenciamos uma relevante redução na espessura muscular do RF, VI e espessura total do quadríceps. Observamos uma alteração na composição da fibra muscular do RF, entretanto este comportamento não foi evidenciado no músculo VI.

Palavras-chave: Ultrassonografia. Músculo quadríceps. Músculo esquelético. Unidades de Terapia Intensiva.

\begin{abstract}
Introduction: Due to the several damages caused by immobility in the bed, it is necessary to have an early assessment of the muscular state of patients.

Objective: Compare ultrasounds and correlate the muscle thickness with the echo intensity of the rectus femoris (RF) and vastus intermedia (VI) muscle in 48 hours and with 7 days of invasive mechanical ventilation (IMV).

Methods: This is a prospective observational study, with 53 images obtained in Intensive Care Unit.

Results: There was a reduction in the muscular thickness of RF, VI and total thickness. We observed a negative correlation between muscle thickness and RF echo intensity in the first 48 hours and 7 days after IMV. Regarding VI, we did not observe an association of VI thickness with the echo intensity in the same period.

Conclusion: We evidenced a relevant reduction in the muscular thickness of the RF, VI and total thickness of the quadriceps. We observed a change in the composition of the RF muscle fiber, however this behavior was not noticed in the VI muscle.
\end{abstract}

Keywords: Ultrasonography. Quadriceps muscle. Muscle, Skeletal. Intensive Care Units.

\section{Cite como}

Vancouver

Lima, RVSA, Fernandes, JS, Sousa, ASL, Lima, DT, Rodrigues, AF, Pedrosa R, Lima, JHM, França, EET Análise ultrassonográfica do quadríceps femoral de pacientes críticos sob ventilação mecânica. Conscientiae Saúde 2020;19(1):1-14, e17090. https://doi.org/10.5585/conssaude.v19n1.17090. 


\section{Introdução}

Anualmente, vários pacientes são admitidos nas unidades de terapia intensiva (UTI), por motivos diversos, e a grande maioria faz uso da ventilação mecânica invasiva (VMI) com necessidade de se manter por um período de restrição ao leito ${ }^{1}$. Essa restrição, caracterizada como imobilidade, pode atingir diversos órgãos e sistemas, causando várias complicações que influenciam na recuperação funcional do paciente, dentre as quais a atrofia muscular ${ }^{2,3}$. Essa surge pelo desuso, uma vez que o sistema muscular é um dos mais moldáveis do corpo humano e mais propensos à lei do uso e desuso, por isso, muito atingido pela restrição ao leito ${ }^{4}$. Nesse processo de desgaste muscular, ocorre a diminuição de volume e função do tecido muscular, de forma precoce e rápida nos primeiros dias de internação na $\mathrm{UTI}^{5,6}$. Kasper et al. demonstraram que 4 horas de restrição ao leito podem proporcionar alterações deletérias nas estruturas musculares 7. Essa perda de massa muscular representa, além de um achado precoce da fase aguda, um preditor de comprometimento funcional, retardando o desmame, o que aumenta a permanência hospitalar, bem como incapacidade funcional aguda e de longo prazo ${ }^{1,5,8}$.

Frente aos diversos danos gerados pela imobilidade no leito, faz-se necessária uma avaliação precoce do estado muscular dos pacientes em UTI. Muitos são os métodos propostos para essa avaliação, porém são desafiadores na rotina, devido à necessidade de profissionais treinados, de cooperação ativa dos pacientes, cuja maioria encontra-se sedada, e da natureza invasiva desses métodos, que são de alto custo e de pouca confiabilidade, e muitos não-viáveis e/ou de difícil acesso ${ }^{9}$. Nesse cenário, as análises por imagens podem ser um método mais acessível, porém nem todos os equipamentos são de fácil utilização, e nem estão disponíveis em ambientes de $\mathrm{UTI}^{5}$. Assim, a ultrassonografia muscular aparece como um método clinicamente viável, facilmente aplicável, não-invasivo, de baixo custo, com um grande potencial de avaliar espessura e estrutura musculares de pacientes restritos ao leito, sedados ou não ${ }^{6,10-12}$.

As imagens ultrassonográficas descritas na literatura apontam para o aumento na ecointensidade muscular. Esse aumento está associado à diminuição da força muscular e da capacidade funcional durante o despertar de pacientes criticamente enfermos, por isso, é sugerido como marcador de prognóstico enquanto o paciente não é capaz de cooperar com testes funcionais volitivos ${ }^{5}$.

A análise do músculo quadríceps femoral por ultrassom é bem descrita pela literatura, que aponta esse músculo como o mais propenso à atrofia precoce, comparativamente aos músculos do membro superior ${ }^{13}$. Estudos anteriores mostram que o compartimento anterior 
apresenta maiores reduções de massa muscular em menor tempo, assim, se faz uma boa escolha para avaliação de massa muscular do reto femoral (RF) e vasto intermédio (VI), já que ocupam a maior parte do compartimento anterior da $\operatorname{coxa}^{14}$, e são importantes extensores de joelho e flexores de quadril simultaneamente no processo de sentar e levantar ${ }^{4}$.

Os objetivos deste estudo foram comparar as imagens ultrassonográficas das fibras do RF e VI do músculo quadríceps em até 48 horas e com 7 dias de VMI, em pacientes críticos, e correlacionar a espessura muscular com os achados de ecointensidade do músculo quadríceps em até 48 horas e com 7 dias de VMI.

\section{Métodos}

Delineamento do estudo: estudo observacional prospectivo de centro único, realizado em uma UTI clínica na cidade de João Pessoa, no Estado da Paraíba, Brasil. A aprovação ética institucional foi emitida pelo Comitê de Ética e Pesquisa (CEP) do Hospital Universitário Lauro Wanderley (HULW), por meio do parecer de número 3.283.172. Em conformidade com a Declaração de Helsinki, foi exigido o consentimento, informado por escrito pelo responsável legal pelo paciente, para participação no estudo.

Triagem e elegibilidade: o grupo de estudo foi selecionado através de amostragem por conveniência dos pacientes internados na UTI, ao longo do período de julho de 2019 a fevereiro de 2020. Neste período todos os pacientes internados na referida UTI e que estavam com até 48 horas ou 7 dias de VMI foram incluídos na pesquisa; um pesquisador frequentava a UTI diariamente no período da tarde e realizava a triagem dos pacientes. Uma vez atendidos os critérios de inclusão, o pesquisador realizava uma avaliação através dos registros médicos, das informações demográficas, da história clínica, do diagnóstico, tempo de internamento na UTI, valor do índice de massa corpórea (IMC) e utilização das seguintes drogas: corticosteroides, bloqueadores neuromusculares, sedativos e drogas vasoativas. Indivíduos com incapacidade de andar sem assistência antes da doença aguda na UTI, gestantes, pacientes com IMC > $35 \mathrm{Kg} / \mathrm{m}^{2}$, doença neuromuscular e vascular previamente diagnosticada, histórico de acidente vascular encefálico e lesões na pele no lugar da avaliação ultrassonográfica foram excluídos. Foram avaliados o nível de mobilização, baseado no protocolo citado por Dantas et al. ${ }^{15}$, e a massa muscular, por ultrassonografia em até 48 horas e com 7 dias de VMI.

Procedimento de imagem por ultrassonografia: um ultrassom (LOGIQ P6, GE Healthcare, Chicago, EUA) foi utilizado com transdutor linear de 5 a $10 \mathrm{MHz}$ para obter imagens nas primeiras 48 horas e no sétimo dia de VMI. As imagens foram obtidas 
transversalmente ao compartimento anterior da coxa direita, com a medida ultrassonográfica em modo $\mathrm{B}^{14}$. As medidas foram obtidas com o paciente em posição supina, Semi-Fowler, com os joelhos completamente estendidos, com rotação neutra do membro inferior ${ }^{16} \mathrm{e}$ os músculos relaxados. O transdutor foi colocado perpendicularmente ao eixo do membro, localizado a dois terços do caminho entre a espinha ilíaca ântero-superior e o côndilo lateral do joelho ${ }^{17}$, marcado por um traço feito com caneta marcadora. $\mathrm{O}$ gel de acoplamento foi aplicado em abundância para minimizar a distorção gerada pelos tecidos subjacentes ${ }^{18}$. A espessura do RF e do VI foi capturada, pelo avaliador, através da visão lateral da coxa, com o mínimo de pressão sobre a pele. A imagem foi logo congelada para armazenamento na memória interna do equipamento, a fim de transferi-la, em seguida, para o computador de análise. Os mesmos pontos e posicionamento foram utilizados nos dois momentos da avaliação. Esta medida tem alta confiabilidade intra e interexaminador ${ }^{12,19}$. Para cada imagem obtida, o transdutor foi localizado perpendicularmente à perna direita do paciente. Dessa imagem foram realizadas as análises que seguem.

Imagem anterior do RF, VI e espessura total: localização da linha reta para quantificação da espessura do VI, iniciando-se no ponto mais alto do fêmur e encerrando-se no final da espessura do músculo, excluindo a fáscia muscular (Figura 1A); localização da linha reta para quantificação da espessura de RF, iniciando-se no ponto mais central, excluindo as suas fáscias (Figura 1B), e localização do traçado partindo do ponto mais alto do fêmur até o final da fáscia de reto femoral, para quantificação da espessura total (ET) do quadríceps femoral (Figura 1B).

Figura 1A e 1B - Imagem de ultrassonografia anterior demonstrando a espessura do vasto intermédio (VI) (1A) e reto femoral (RF) (1B) e espessura total (1B), respectivamente

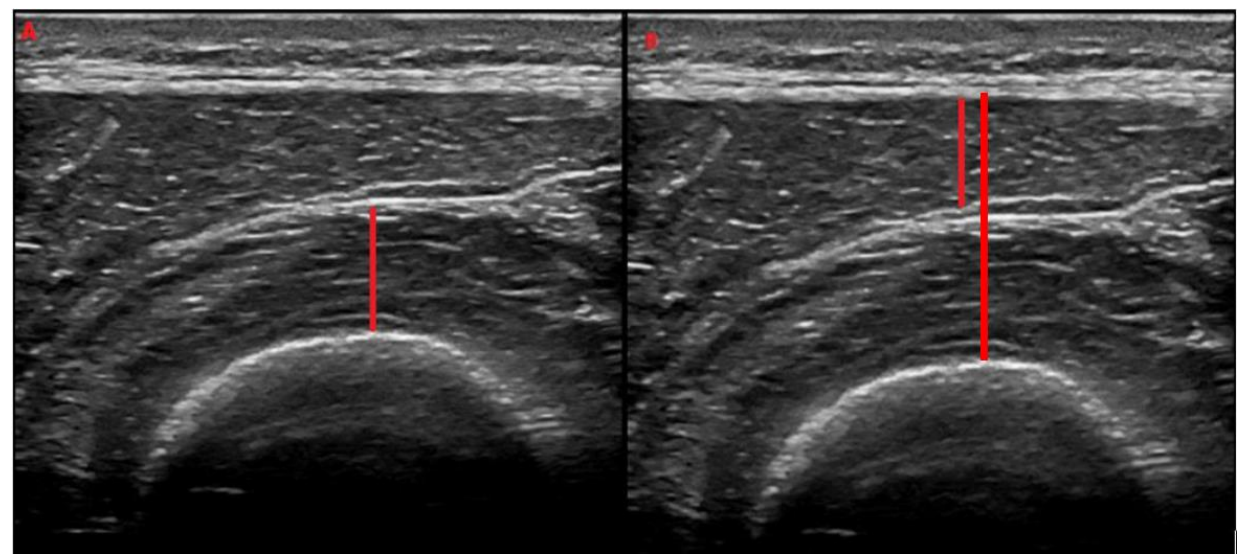

Fonte: Autores. 
(1) Imagem anterior da região de interesse (ROI): posicionamento da ROI pelo método quadrado na região mais volumosa do músculo RF, para análise da ecointensidade (Figura 2).

Figura 2 - Imagem de ultrassonografia anterior demonstrando a região de interesse (ROI) do músculo reto femoral (RF)

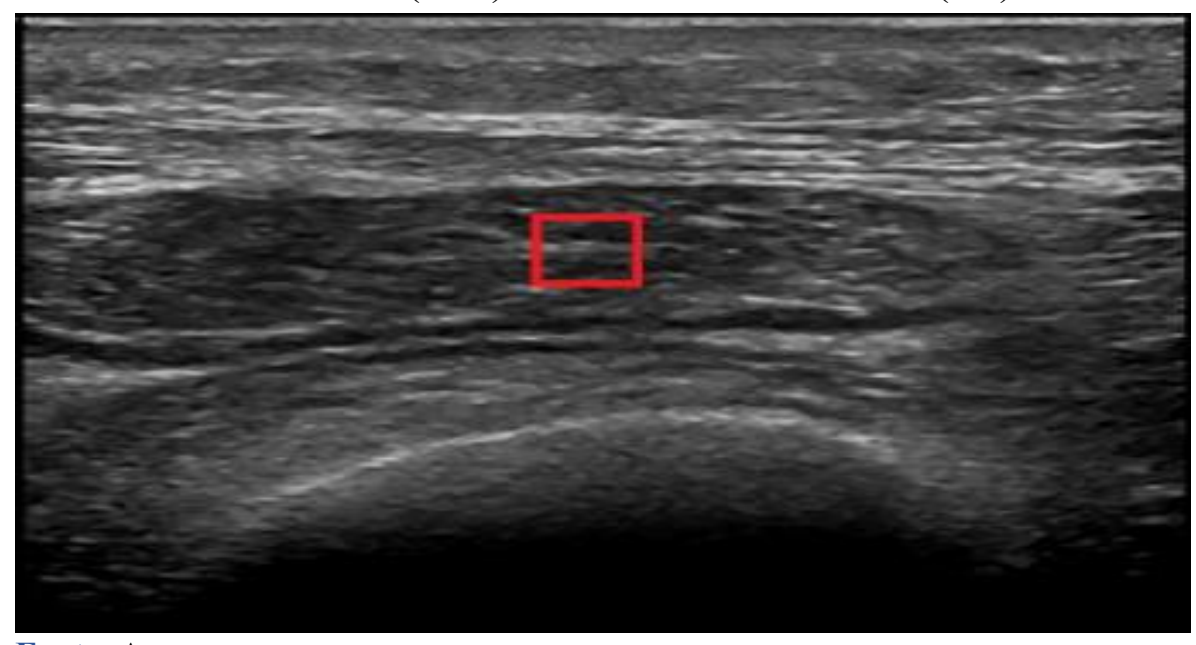

Fonte: Autores.

A análise de ecointensidade foi dada através da escala de cinza, após ser determinada a ROI pelo método quadrado ${ }^{9}$, citado pela literatura como um dos melhores métodos para análises de músculos esqueléticos e com maior confiabilidade ${ }^{5,17}$. A ROI foi composta por $50 \mathrm{~mm}$ de altura e de largura - valor determinado devido à arquitetura muscular que os pacientes apresentavam e localizado na região mais volumosa dos músculos ${ }^{5}$. Após o posicionamento da ROI, foram escolhidas as opções desejadas, para assim medir-se e serem gerados os valores, que foram dados em pixels pelo histograma da escala de cinza, a qual varia de 0 (= preto) a 255 (= branco). Quanto mais alto o valor mais clara estava a imagem, o que aponta um provável infiltrado no músculo e/ou depleção de miofibrilas ${ }^{20}$. As imagens foram salvas no disco rígido do ultrassom e transferidas para análises adicionais em um computador, usando-se o software ImageJ (NIH, Bethesda, MD) ${ }^{16}$, calibrado e cada pixel equivalendo a $1 \mathrm{~mm}$.

Tamanho da amostra e análises estatísticas: um tamanho de amostra pragmático de 22 pacientes foi identificado. Para calcular o tamanho da amostra, foi utilizado o programa WinPepi (PEPI-for-Windows), considerando-se os seguintes critérios: intervalo de confiança de $95 \%$, desvio padrão de $20 \%$, com erro e perda amostral de $5 \%$ e $20 \%$, respectivamente, com base no estudo de Parry et al..$^{5}$ que submeteram 22 pacientes a uma avaliação de ultrassonografia de quadríceps para analisar a massa e qualidade muscular de pacientes críticos.

Para análise estatística, primeiramente, foi utilizado o teste de Kolmogorov-Smirnov e o teste Shapiro-Wilk para identificar a normalidade dos dados. Considerando-se que as variáveis foram não-paramétricas, foi utilizado o Coeficiente de Correlação de Spearman para 
analisar a relação entre a espessura muscular e os achados de ecointensidade do músculo quadríceps. Foi utilizado o programa SPSS, na versão 25.0, com nível de significância de 5\%.

\section{Resultados}

Durante o período do estudo, de julho de 2019 a fevereiro de 2020, foram admitidos 230 pacientes na UTI, dos quais apenas 59 atenderam aos critérios estabelecidos para participarem do estudo e 171 apresentaram os critérios de exclusão. Dos 59 pacientes, 33 foram avaliados em até 48 horas e 26 em 7 dias de VMI. Dos 33 avaliados em até 48 horas, dois (02) não apresentavam uma área mínima para a avaliação da ROI dos músculos VI e RF e dois (02) apresentaram imagens insuficientes para a realização de análises. Já dos 26 avaliados em 7 dias, dois (02) não apresentavam uma área mínima para a avaliação da ROI dos músculos VI e RF. Portanto, foram analisados $29(n=29)$ em até 48 horas e $24(n=24)$ em 7 dias.

A Tabela 1 mostra a caracterização da amostra. Observa-se que o número de mulheres é superior a 50\% em ambos os grupos com 48 horas e 7 dias, sendo maior no grupo com 7 dias de internamento (79,9\%). Em relação à faixa etária, o número de pessoas entre 41-65 anos de idade avaliados em 48 horas constitui a maioria, com 38\% dos indivíduos, já a população acima de 65 anos se sobressai com 37,5\%, no grupo com 7 dias de internação.

O modo de ventilação mecânica revela que, nas primeiras 48 horas, todos os pacientes estavam em VMI, porém, após 7 dias de a terem iniciado, 37\% dos pacientes não se encontravam mais em VMI. Quanto ao nível de mobilização, observamos que $70 \%$ dos indivíduos de ambos os grupos estavam com nível I nos dois períodos avaliados.

Em relação à presença da sedação, no grupo de até 48 horas de VMI, 82,8\% estavam sedados, comparando-se a 70,9\% após 7 dias de iniciada a VMI. No que se refere ao diagnóstico principal, observamos que 33,3\% da população avaliada em até 48 horas de VMI são de pacientes no pós-operatório, enquanto que com 7 dias, temos apenas 12,5\% desta população, com predomínio de pacientes clínicos, $87,5 \%$, neste período. 
Tabela1 - Caracterização da amostra, com o número de pacientes e percentual, em até 48 horas de VMI e 7 dias após sua introdução

\begin{tabular}{|c|c|c|}
\hline & n $(\%)-48 h$ & n (\%) - 7 dias \\
\hline \multicolumn{3}{|l|}{ Sexo } \\
\hline Mulheres & $15(51,0)$ & $17(70,9)$ \\
\hline Homens & $14(49,0)$ & $7(29,1)$ \\
\hline Total & $29(100,0)$ & $24(100,0)$ \\
\hline \multicolumn{3}{|l|}{ Idade (anos) } \\
\hline $18-40$ & $9(31,0)$ & $8(33,4)$ \\
\hline $41-65$ & $11(38,0)$ & $7(29,1)$ \\
\hline $66-100$ & $9(31,0)$ & $9(37,5)$ \\
\hline \multicolumn{3}{|c|}{ Modo de Ventilação Mecânica } \\
\hline PCV & $23(79,3)$ & $11(45,8)$ \\
\hline PSV & $6(20,7)$ & $4(16,7)$ \\
\hline $\mathrm{RE}$ & - & $9(37,5)$ \\
\hline Total & $29(100,0)$ & $24(100,0)$ \\
\hline \multicolumn{3}{|l|}{ Nível de Mobilização } \\
\hline I & $25(86,2)$ & $18(75,0)$ \\
\hline II & $3(10,3)$ & $4(16,3)$ \\
\hline III & $1(3,4)$ & $2(8,3)$ \\
\hline IV & - & - \\
\hline $\mathrm{V}$ & - & - \\
\hline Total & $29(100,0)$ & $24(100,0)$ \\
\hline \multicolumn{3}{|l|}{ Sedado } \\
\hline Sim & $24(82,8)$ & $17(70,9)$ \\
\hline Não & $5(17,7)$ & $7(29,1)$ \\
\hline Total & $24(100,0)$ & $24(100,0)$ \\
\hline \multicolumn{3}{|l|}{ Diagnóstico Principal } \\
\hline Paciente clínico & $20(66,7)$ & $21(87,5)$ \\
\hline Desordem cardíaca & $3(11,1)$ & $2(9,5)$ \\
\hline Desordem respiratória & $4(16,6)$ & $4(19,0)$ \\
\hline Desordem renal & $5(27,7)$ & $2(9,5)$ \\
\hline Desordem neurológica & $2(1,11)$ & $2(9,5)$ \\
\hline Sepse & $6(33,3)$ & $7(33,3)$ \\
\hline Paciente pós-operatório & $9(33,3)$ & $3(12,5)$ \\
\hline Total $P$ P & $29(100,0)$ & $24(100,0)$ \\
\hline
\end{tabular}

Legenda: VMI: ventilação mecânica invasiva, PCV: ventilação com pressão controlada, PSV: ventilação por pressão de suporte, RE: respiração espontânea.

Fonte: Autores.

A Tabela 2 mostra média, desvio padrão, mediana e intervalo mínimo e máximo da medida de espessura dos músculos VI, RF e a ET do grupo muscular do quadríceps. Observamos que tanto a média como a mediana, no grupo de 7 dias após da introdução da VMI, são menores do que o grupo de 48 horas, nos três segmentos avaliados. 
Tabela 2 - Estatística descritiva da medida de espessura do vasto intermédio, reto femoral e espessura total do avaliados em até 48 horas de ventilação mecânica invasiva e após 7 dias de sua introdução

\begin{tabular}{ccc}
\hline Músculo quadríceps & $\mathbf{4 8 h}(\mathbf{n}=\mathbf{2 9})$ & $\mathbf{7}$ dias $\mathbf{( n = 2 4 )}$ \\
\hline Vasto intermédio & $97,93 \pm 33,76$ & $84,33 \pm 26,29$ \\
Média \pm DP $(\mathrm{mm})$ & $93,02(54,0-192,0)$ & $85,00(50,1-154,0)$ \\
Mediana (min-max) (mm) & \\
Reto femoral & $94,52 \pm 25,54$ & $89,79 \pm 30,04$ \\
Média \pm DP $(\mathrm{mm})$ & $90,00(52,0-151,0)$ & $86,50(51,0-162,0)$ \\
Mediana (min-max) (mm) & & \\
Espessura total & $195,97 \pm 48,74$ & $177,88 \pm 49,53$ \\
Média \pm DP $(\mathrm{mm})$ & $193,00(112,0-325,0)$ & $177,50(106,0-266,0)$ \\
Mediana \pm (min-max) $(\mathrm{mm})$
\end{tabular}

Legenda: $\mathrm{DP}=$ desvio padrão; $\mathrm{mm}=$ milímetros; $\mathrm{min}=$ mínimo valor; $\max =$ máximo valor. Fonte: Autores.

A Tabela 3 mostra média, desvio padrão, mediana e intervalo mínimo e máximo da média da escala de cinza dos músculos VI e RF. Observamos que tanto a média, como a mediana de ambos os músculos, no grupo de 7 dias após a introdução da VMI, são maiores do que no grupo de 48 horas.

Tabela 3 - Estatística descritiva da média de cinza do vasto intermédio e reto femoral em até 48 horas e após 7 dias da introdução da ventilação mecânica invasiva

\begin{tabular}{|c|c|c|}
\hline Músculo quadríceps & 48h $(n=29)$ & 7 dias $(n=24)$ \\
\hline \multicolumn{3}{|l|}{ Vasto Intermédio } \\
\hline Média \pm DP (píxeis) & $91499,55 \pm 32002,425$ & $119768,17 \pm 44143,42$ \\
\hline Mediana (min-max) (píxeis) & $88633,00(34170-171603)$ & $116069,50(50686-202458)$ \\
\hline \multicolumn{3}{|l|}{ Reto Femoral } \\
\hline Média \pm DP (píxeis) & $86771,90 \pm 5560,755$ & $122463,58 \pm 7980,983$ \\
\hline Mediana (mín-max) (píxeis) & $86597,00(23067-154882)$ & $136019,50(56484-192648)$ \\
\hline
\end{tabular}

Na Figura 3A e 3B, observamos uma correlação da espessura com a média da escala de cinza do RF em até 48h e 7 dias após introdução da VMI. Essa correlação é negativa (r= -0,062) em 48 horas e $(r=-0,154)$ em 7 dias, respectivamente, considerada fraca e inversamente proporcional, mostrando que, quanto maior a espessura de RF, menor é a média de escala de cinza. 
Figura 3A e 3B - Gráfico de dispersão simples da espessura por média da escala de cinza do músculo reto femoral em até 48 horas e 7 dias após a introdução de ventilação mecânica invasiva
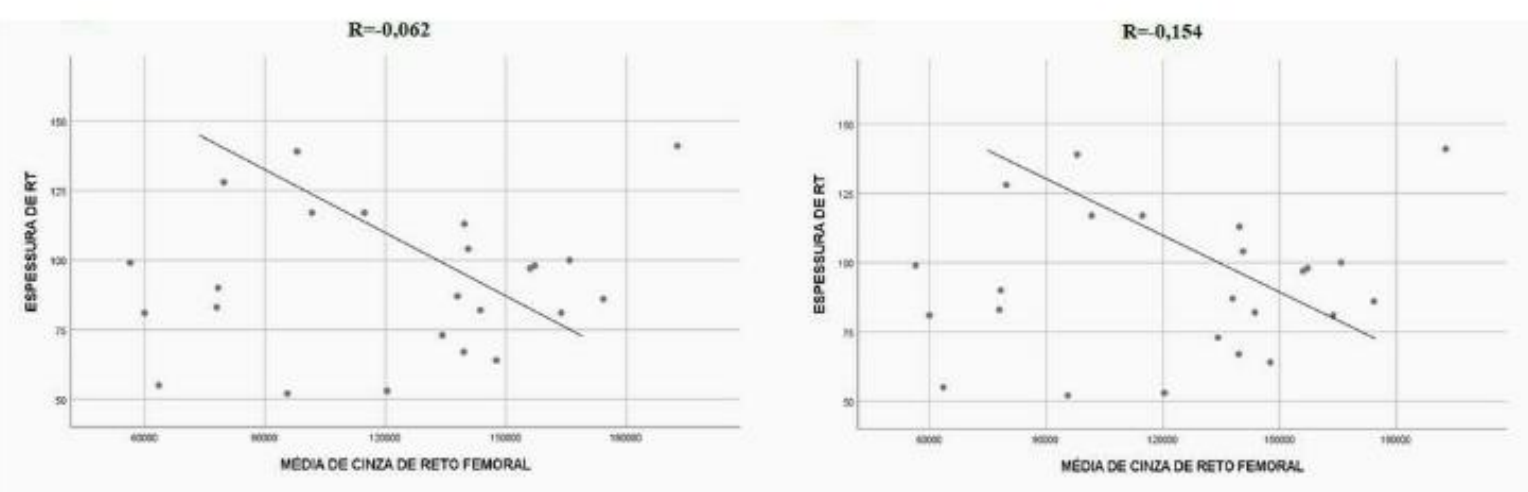

Fonte: Autores.

Em relação à correlação da espessura com a média da escala de cinza do VI em até 48 horas da VMI, essa correlação é positiva $(r=0,314)$, considerada fraca e diretamente proporcional, ou seja, à medida que espessura de VI aumenta, a média da escala de cinza do mesmo músculo aumenta proporcionalmente. Já quando analisada essa correlação com 7 dias da introdução da VMI, observamos que não houve correlação entre essas variáveis $(r=0,012)$, indicando que não há correlação entre as mesmas.

\section{Discussão}

O presente estudo demonstrou que, com o passar do tempo, os pacientes internados na UTI, que foram submetidos à VMI, apresentaram uma redução na espessura total do quadríceps, assim como nos músculos RF e VI isoladamente. Nossos resultados estão em concordância com a literatura onde é bem elucidada a redução na espessura de quadríceps femoral, frente ao avanço do período de internação ${ }^{1,5,21}$, assim como nos músculos isolados ${ }^{5,14,22}$. Ozdemir et al. ${ }^{23}$ apontaram que essa redução na espessura muscular foi mais evidente nos pacientes críticos, quando estes apresentavam um maior risco nutricional. Estudos que analisaram a área de seção transversa do RF afirmam que o mesmo apresentou maior redução quando os indivíduos foram submetidos à $\mathrm{VMI}^{24}$. Alteração na área de seção transversa no RF ao longo de 7 dias correlacionou-se moderadamente com a força após uma semana de admissão na $\mathrm{UTI}^{22}$.

Parry et al. ${ }^{5}$, ao analisarem os valores de ecointensidade de 22 pacientes internos na UTI, observaram um aumento de $12,7 \%$ para VI e 25,5\% para RF com o passar dos dias de internação. Em nosso estudo, foram encontrados resultados semelhantes, pois a média da escala de cinza foi maior em 7 dias após a instalação da VMI do que em 48 horas. Esses resultados demonstram que, com o tempo, a escala de cinza tem valores mais altos, ou seja, mais próximos 
ao branco, uma imagem definida como hiperecoica, caracterizando uma provável lesão da fibra muscular.

Pillen ${ }^{10}$ e Cartwright et al. ${ }^{25}$ explicaram os aspectos musculares identificados pelo ultrassom, em que os músculos normais têm aspecto hipoecoico, pois são relativamente mais pretos por terem baixa ecointensidade. Assim, os músculos saudáveis têm pouca quantidade de tecido fibroso, já em condições patológicas, quando o tecido muscular é substituído por tecido adiposo, fibroso e outros, as imagens ultrassonográficas apresentam-se de forma hiperecoica, resultando em transições com impedância acústica diferente e muita reflexão do feixe de ultrassom. Assim, as imagens apresentam colorações mais brancas e valores maiores na escala de cinza, com imagens do ultrassom apresentando limites musculares claramente visíveis, pois a imagem do epimísio é altamente reflexiva, assim como os ossos em indivíduos normais são distintos com uma sombra óssea anecoica por baixo, por apresentar eco forte e distinto. Esse aumento na escala de cinza ainda pode inferir necrose muscular e infiltração de tecido gorduroso em substituição às fibras musculares.

Podemos assim afirmar, com base nos achados literários e nos resultados do presente estudo, que com o passar do tempo os pacientes apresentam presença de infiltrados musculares com deterioração muscular. No estudo de Parry et al..$^{5}$, a ecointensidade também apresenta relação com a força e a funcionalidade dos pacientes após o despertar na UTI, pois os mesmos que apresentaram valores na escala de cinza mais altos demonstraram força e funcionalidade mais debilitadas.

Na correlação entre a espessura do músculo e a média da escala de cinza do RF, nossos resultados mostram que há uma associação negativa, ou seja, quando a espessura diminui a média da escala de cinza aumenta, assim acreditamos que o músculo está perdendo espessura e sofrendo, concomitantemente, alterações na composição de sua fibra. Nossos resultados corroboram os estudos de Katari et al. ${ }^{14}$ que, analisando o músculo RF, observaram uma redução considerável de sua espessura em internos na UTI, assim como os estudos de VallsMatarín et al. ${ }^{26}$ que também encontraram uma redução da espessura do RF, atribuída à variável tempo de internação. Puthucheary et al. ${ }^{27}$, que correlacionaram a biópsia e ecointensidade e apresentaram a composição do músculo do quadríceps femoral nas primeiras semanas da doença grave, observaram um aumento da ecointensidade, indicando a presença de necrose nas miofibras, assim como edema, infiltrados de neutrófilos e deposição de fibrina, com a presença de células ricas em macrófagos, sendo evidenciado uma fasceíte que se entendia mais profundamente no músculo, detectado pela biópsia. 
Diferentemente do RF, o comportamento do VI nas primeiras 48 horas de VMI, em nosso estudo, demonstrou uma correlação positiva entre a espessura desse músculo com a média da escala de cinza, ou seja, quanto maior a espessura do músculo maior foi a média de escala de cinza, se aproximando mais do branco, isso mostra que uma maior espessura desse músculo não pode ser traduzida em uma melhor composição de sua fibra muscular. Nossos achados são semelhantes aos do estudo de Lopez et al. ${ }^{28}$ realizado com senhoras ativas, o qual demonstra que a ecointensidade do quadríceps femoral pode estar aumentada enquanto a espessura aumenta ou se mantém, pois os infiltrados musculares podem camuflar essa redução de espessura. Assim, acreditamos que ocorra o mesmo com os pacientes críticos, como citado anteriormente: eles apresentam edema e infiltrados e o aumento na ecointensidade pode referir mudanças na composição da fibra muscular. Valls-Matarín et al. ${ }^{26}$ mostraram que pacientes em sua alta hospitalar tinham pesos próximos aos de sua admissão e redução significativa da massa muscular, sendo o peso corpóreo também considerado não preditor da composição da fibra muscular. Já após 7 dias da introdução da VMI, não observamos correlação entre a espessura muscular com a ecointensidade do VI, ou seja, não houve nenhum tipo de associação entre essas variáveis neste período. Esse achado pode estar relacionado a uma retardada diminuição da espessura muscular do VI em comparação com o $\mathrm{RF}^{5}$, pois, por tratar-se de um músculo mais profundo, esta perda torna-se um pouco menos evidente nos primeiros dias de internamento. Em consequência, o maior tempo de internação favorece a maior necrose de miofibras e regeneração muscular, o que pode estar associado ao aumento da ecointensidade neste período $^{27}$.

Este comportamento diferente, observado entre espessura muscular e a média de escala de cinza de RF e VI, pode ser explicado por Moore et al. ${ }^{29}$, que relatam que esses músculos têm distribuições de fibras diferentes em suas composições, pois o RF é descrito como músculo de força, que auxilia em movimentos rápidos, biarticular, composto principalmente por fibras do tipo II ou fibras de contração rápida. Já o VI é descrito como músculo estabilizador, importante no equilíbrio, no ortostatismo e marcha, tratando-se de músculo não articular e composto principalmente por fibras de resistência ou do tipo I. Essa composição diferente da fibra muscular pode também interferir, comparativamente, numa maior perda desses músculos ${ }^{27}$.

Embora tenha obtido resultados positivos, nosso estudo apresentou algumas limitações relacionadas à obtenção das imagens na fase aguda da doença, a alta e a óbitos no período do estudo, ausência de profissional habilitado para obtenção das imagens no respectivo dia da avaliação e indisponibilidade do equipamento de ultrassom. Algumas dessas limitações também foram enfrentadas por Palakshappa et al. ${ }^{22}$. 


\section{Conclusão}

Os resultados do presente estudo apontam para as seguintes conclusões: ${ }^{1}$ houve uma redução na espessura dos músculos RF, VI e ET do quadríceps, quando comparamos as primeiras 48 horas com 7 dias após a introdução da VMI; ${ }^{2}$ o aumento da ecointensidade, evidenciado pelo aumento da média da escala de cinza, demonstra que quanto maior o tempo de internação mais alterações na composição das fibras são evidenciadas nos pacientes; $\mathrm{e}^{3}$ há uma correlação negativa da espessura muscular com a ecointensidade do músculo RF, não evidenciada no músculo VI.

Com base nos resultados deste estudo, recomendamos que pesquisas futuras se voltem para a mensuração da espessura e ecointensidade do RF e VI em estudos ultrassonográficos; e para os preditores mais importantes de resultado funcional, morbidade e mortalidade por ultrassonografia. Torna-se necessário, assim, um exame combinado de ultrassonografia com investigações laboratoriais para examinar os mecanismos moleculares envolvidos.

Finalmente, é fundamental identificar indivíduos com maior risco precoce para permitir a investigação de estratégias de intervenção, que podem ser aplicadas precocemente para tentar minimizar essas alterações e suas consequências funcionais deletérias.

\section{Referências}

1. Hernández-Socorro CR, Saavedra P, López-Fernández JC, Ruiz-Santana S. Assessment of Muscle Wasting in Long-Stay ICU Patients Using a New Ultrasound Protocol.Nutrients.2018 Dec 01; 10(12):1849-59. https://doi.org/10.3390/nu10121849.

2. Aquim EE, Bernardo WM, Buzzini RF, Azeredo NSG, Cunha LS, Damasceno MCP, et al. Diretrizes Brasileiras de Mobilização Precoce em Unidade de Terapia Intensiva. Rev Bras Ter Intensiva. 2020 jan 20; 31(4):434-43. https://doi.org/10.5935/0103-507x.20190084.

3. Toptas M, Yalcin M, Akkoc I, Demir E, Metin C, Savas Y, et al. The Relation between Sarcopenia and Mortality in Patients at Intensive Care Unit. BioMed Research International. 2018 fev 12; 2018:ID 5263208: 1-9. https://doi.org/10.1155/2018/5263208.

4. Gruther W, Benesch T, Zorn C, Paternostro-Sluga T, Quittan M, Fialka-Moser V, et al. Muscle wasting in intensive care patients: Ultrasound observation of the M. quadriceps femoris muscle layer. J Rehabil Med 2008; 40 (3):185-9. https://doi.org/10.2340/16501977-0139.

5. Parry SM, El- Ansary D,Cartwright MS, Sarwal A, Berney Koopman R. Ultrasonography in the intensive care setting can be used to detect changes in the quality and quantity of muscle and is related to muscle strength and function. Journal of Critical Care. 2015 Jun 3; 30(5):1151.e9-14. https://doi.org/ 10.1016/j.jcrc.2015.05.024. 
6. Pardo E, Behi HE, Boizeau P, Verdonk F, Alberti C, Lescot T. Reliability of ultrasound measurements of quadriceps muscle thickness in critically ill patients. BMC Anesthesiology.2018 Dec 27; 12(205):1-8. https://doi.org/10.1186/s12871-018-0647-9.

7. Kasper CE, Talbot L, Gaines JM. Skeletal Muscle Damage and Recovery. AACN Adv Crit Care 2002 May; 13(2):237-47. https://doi.10.1097/00044067-200205000-00009.

8. Desai SV, Law TJ, Needham DM. Long-term complications of critical care. Crit Care Med 2011; 39(2):371-9. https://doi.org/10.1097/ccm.0b013e3181fd66e5.

9. Sarwal A, Parry SM, Berry MJ, Hsu FC, Lewis MT, Justus NW et al. Interobserver Reliability of Quantitative Muscle Sonographic Analysis in the Critically Ill Population. J Ultrasound Med. 2015 Jul; 34(7):1191-200. https://doi: 10.7863/ultra.34.7.1191.

10. Pillen S. Skeletal muscle ultrasound. European Journal Translational Myology 2010; 1(4):145-155. https://doi.org/10.4081/ejtm.2010.1812.

11. Vieira L, Rocha LPB, Mathur S, Santana L, Melo FP, Silva VZM, et al. Reliability of Skeletal Muscle Ultrasound in Critically Ill Trauma Patients. Rev Bras Ter Intensiva. 2019 OctDec; 31(4), 464-73. https://doi.org/10.5935/0103-507X.20190072.

12. Paris MT, Mourtzakis M, Day A, Leung R, Watharkar S, Kozar R. Validation of Bedside Ultrasound of Muscle Layer Thickness of the Quadriceps in the Critically Ill Patient (VALIDUM Study). JPEN J Parenter Nutr. Enteral. 2016 Mar 16; 41(2):171- 80. https://doi: $10.1177 / 0148607116637852$.

13. Turton P, Hay R, Taylor J,McPhee J, Welters I. Human limb skeletal muscle wasting and architectural remodeling during five to ten days intubation and ventilation in critical care - an observational study using ultrasound. BMC Anesthesiol. 2016 Nov 29; 16(1):119. https://doi.org/10.1186/s12871-016-0269-z.

14. Katari Y, Srinivasan R, Arvind P, Hiremathada S. Point-of-Care Ultrasound to Evaluate Thickness of Rectus Femoris, Vastus Intermedius Muscle, and Fat as an Indicator of Muscle and Fat Wasting in Critically Ill Patients in a Multidisciplinary Intensive Care Unit. Indian J Crit Care Med. 2018 Nov; 22(11):781-8. https://doi.org/10.4103/ijccm.IJCCM_394_18.

15. Dantas CM, Silva PFS, Siqueira FHT, Pinto RMF, Matias S, Maciel C, et al. Influência da mobilização precoce na força muscular periférica e respiratória em pacientes críticos. Rev Bras Ter Intensiva. 2012 jun; 24(2): 173-78. https://doi.org/10.1590/S0103-507X2012000200013.

16. Weinel LM, Summers MJ, Chapple LA. Ultrasonography to measure quadríceps muscle in critically ill patients: A literature review of reported methodologies. Anaesth Intensive Care. 2019 Sep; 47(5):423-34. https://doi: 10.1177/0310057X19875152.

17. Silva PE, Carvalho KL, Melo L, Vieira L. Ultrassonografia musculoesquelética - Bases teóricas para avaliação da arquitetura muscular em pacientes criticamente enfermos. In: Associação Brasileira de Fisioterapia Cardiorrespiratória e Fisioterapia em Terapia intensiva; Martins JÁ, Reis LFF, Andrade FMD, organizadores. PROFISIO Programa de atualização em Fisioterapia em Terapia intensiva Adulto: Ciclo 8. Porto Alegre: Artmed Pan-americana: 2018. p.73-120. 
18. Annetta MG, Pittiruti M, Silvestri D, Grieco DL, Maccaglia A, La Torre MF, et al. Ultrasound assessment of rectus femoris and anterior tibialis muscles in young trauma patients. Ann Intensive Care. 2017 Oct 6; 7(1):104-12. https://doi.org/10.1186/s13613- 017-0326-X.

19. Toledo DO, Silva DCL, Santos DM, Freitas BJ, Dib R, Cordioli RL, et al. Ultrassom à beira do leito como ferramenta prática para avaliação da massa muscular. Rev Bras Ter Intensiva. 2017; 29(4):476-80. https://doi.org/10.5935/0103-507X.20170071.

20. Grimm A, Teschner U, Porzelius C, Ludewig K, Zielske J, Witte OW, et al. Muscle ultrasound for early assessment of critical illness neuromyopathy in severe sepsis. Crit Care. 2013 oct 7; 17(5): R227. https://doi.org/10.1186 / cc13050.

21. Rodrigues CN. Ultrassonografia na avaliação e no seguimento nutricional de pacientes críticos [dissertação]. Universidade Federal de Minas Gerais; 2019.

22. Palakshappa JA, Reilly JP, Schweickert WD, Anderson BJ, Khoury V Shashaty, MG, et al. Quantitative peripheral muscle ultrasound in sepsis: Muscle área superior to thickness. J Crit Care. 2018 Oct; 47: 324-30. https://doi: 10.1016/j.jcrc.2018.04.003.

23. Özdemir U, Özdemir M, Aygencel G, Kaya B, Türkoğlu M. The role of maximum compressed thickness of the quadriceps femoris muscle measured by ultrasonography in assessing nutritional risk in critically-ill patients with different volume statuses. The role of maximum compressed thickness of the quadriceps femoris muscle measured by ultrasonography in assessing nutritional risk in ritically-ill patients with diferente volume statuses. Rev Assoc Med Bras. 2019 Apr 19; 65(7):952-58.https://doi.org/10.1590/18069282.65.7.952.

24. Borges RC. Associação entre a degradação muscular e a força muscular em pacientes que desenvolveram [tese]. Faculdade de Medicina da Universidade de São Paulo; 2018.

25. Cartwright MS, Kwayisi G, Griffin lp, Sarwal A, Walker FO, Harris JM, et al. Quantitative neuromuscular ultrasound in the intensive care unit. Muscle \& Nerve. 2013 Feb; 47(2):255-9. https://doi.org/10.1002/mus.23525.

26. Valls-Matarín J, Cotillo-Fuente M, Grané-Mascarell N, Quintana, S. Variación de la masa muscular y el peso en el paciente crítico. Enfermería Intensiva. 2015 Jul 9; 26(3), 86-91. https://doi:10.1016/j. enfi.2015.05.001.

27. Puthucheary ZA, Phadke R, Rawal J, McPhail MJ, Sidhu PS, Rowlerson A. Qualitative Ultrasound in Acute Critical Illness Muscle Wasting. Crit Care Med. 2015 Aug; 43(8):160311. https://doi: 10.1097/CCM.0000000000001016.

28. Lopez P, Radaelli R, Rech A, Wilhelm EM, Pinto RS. Muscle quality, but not muscle thickness, is decreased in different age groups of active older women. Rev Bras Cineantropom Desempenho Hum. May / Jun de 2015; 17(3):347-56. https://doi.org/10.5007/19800037.2015v17n3p347.

29. Moore K, Dalley A, Agur A. Anatomia orientada para a clínica. 7th ed. Rio de Janeiro: Guanabara Koogan; 2014. 